\title{
Componentizando Processos Legados de Software Visando a Reutilização de Processos
}

\author{
Ahilton Silva Barreto ${ }^{1,3}$, Leonardo Gresta Paulino Murta ${ }^{2}$, Ana Regina Rocha ${ }^{1}$ \\ ${ }^{1}$ COPPE/UFRJ - Programa de Engenharia de Sistemas e Computação \\ Caixa Postal 68511 - CEP: 21945-970 - Rio de Janeiro - RJ \\ ${ }^{2}$ Instituto de Computação - Universidade Federal Fluminense (UFF) \\ Niterói - RJ \\ ${ }^{3}$ Banco Nacional de Desenvolvimento Econômico e Social (BNDES) \\ Av. República do Chile, 100 - CEP 20031-917 - Rio de Janeiro, RJ \\ ahilton@cos.ufrj.br, leomurta@ic.uff.br, darocha@cos.ufrj.br
}

\begin{abstract}
This paper presents an approach to adapt pre-existent software processes of an organization (i.e., legacy processes) as a starting point to the adoption of software process reuse, also aiming to ease the implementation of higher maturity levels. This adaptation involves the definition of software process components, features, and process lines, based on legacy processes and legacy process assets. We also present an example of use of parts of our approach, considering some legacy processes from COPPE/UFRJ.
\end{abstract}

Resumo. Este artigo apresenta uma abordagem para adaptação de processos de software existentes em uma organização (processos legados) como uma etapa inicial para a adoção da reutilização de processos visando inclusive facilitar a implementação de níveis mais altos de maturidade. Essa adaptação envolve a definição de componentes, características e linhas de processo a partir dos processos e ativos de processos legados. É apresentado, também, um exemplo de utilização de partes da abordagem proposta, considerando alguns processos legados da COPPE/UFRJ.

\section{Introdução}

Definir um processo de software não é uma atividade simples; exige experiência e envolve o conhecimento de muitos aspectos da engenharia de software. É necessário levar em conta muitos fatores, como: necessidades e características da organização ou projeto, técnicas e métodos que serão utilizados, conformidade com padrões ou modelos de referência e restrições de negócio (prazo, custo, etc). Assim, essa atividade costuma exigir um profissional especializado que consiga harmonizar todos esses fatores.

Como forma de promover a reutilização do conhecimento relacionado a processos de software, técnicas de reutilização têm sido adaptadas do desenvolvimento de produtos de software para o contexto da definição de processos de software [Kellner, 1996; Reis, 2002; Washizaki, 2006]. O propósito é facilitar a definição de processos, diminuindo o custo e o esforço associado à atividade, além de possivelmente aumentar a qualidade dos processos gerados, inclusive tornando a atividade acessível a profissionais menos experientes. Conceitos como componentes, arquiteturas, linhas de 
produtos e padrões têm sido utilizados para a definição e melhoria de processos de software.

Ainda no contexto de reutilização, modelos de maturidade e normas como o CMMI-DEV [Chrissis et al., 2006], o MPS.BR [Softex, 2007] e a ISO/IEC 15504 [Iso/Iec, 2004] estabelecem que, em organizações de maior maturidade, os processos devem ser definidos com base em unidades menores de processo, normalmente chamadas de subprocessos ou elementos de processos. A definição deve, inclusive, considerar a estabilidade histórica e dados de desempenho desses subprocessos ou elementos de processo. Ou seja, um processo de software deve ser definido com base em unidades menores e reutilizáveis, reforçando a idéia de componentização de processos.

No entanto, é possível que organizações que desejem tornar seus processos reutilizáveis, através da utilização de conceitos como componentes, características e linhas de processo, partam de uma situação em que processos definidos a partir de alguma outra abordagem não voltada para a reutilização já existam e sejam executados na organização. Assim, é necessário um procedimento para adaptar esses processos "legados" para a nova estrutura baseada em elementos reutilizáveis. É de se esperar que essa adaptação dos processos contribua para facilitar o alcance das práticas esperadas relacionadas à definição de processos nos níveis mais altos de maturidade, além de potencializar a reutilização de processos.

Neste contexto, o objetivo deste trabalho é descrever uma abordagem para guiar a definição de elementos reutilizáveis de processo de software (i.e., componentes, características, linhas de processo) a partir da adaptação de processos e ativos de processo de software existentes em uma organização. Além disso, contextualizar essa etapa de definição de processos para reutilização dentro de uma abordagem maior de definição de processos de software baseada em reutilização, explicando suas principais características e os conceitos envolvidos.

Este artigo está organizado em cinco seções, incluindo esta introdução. A seção 2 aborda a questão da definição e reutilização de processos de software. A seção 3 apresenta a abordagem de definição de processos baseada em reutilização, na qual este trabalho se insere. Na seção 4 é apresentada a abordagem para componentização dos processos legados. Por fim, a seção 5 apresenta as conclusões e perspectivas futuras deste trabalho.

\section{Definição e Reutilização de Processos de Software}

Processos de software podem apresentar grande complexidade e possibilitar diversas alternativas para execução de suas atividades. Desta forma, um processo de software estabelecido permite que profissionais de engenharia de software possam trabalhar de forma ordenada, possibilitando um melhor entendimento do seu trabalho, bem como de outras atividades executadas por outros membros da mesma equipe [Humphrey, 1989].

\footnotetext{
${ }^{1}$ Quando é usada a expressão "processos legados de software" neste artigo, não nos referimos apenas a processos que deixaram de ser usados ou que estão desatualizados em relação às práticas correntes da organização, mas sim a quaisquer processos de software existentes na organização que precisem passar por adaptações para se tornarem mais adequados à reutilização de processos.
} 
Segundo a norma ISO/IEC 15504 [Iso/Iec, 2004], um processo padrão é o conjunto de definições dos processos básicos que guiam todos os processos em uma organização. Podem ser definidos em múltiplos níveis em uma organização e podem ser relacionados de maneira hierárquica. Um processo definido, por sua vez, é um processo que é gerenciado (planejado, monitorado e ajustado), e adaptado a partir do conjunto de processos padrão da organização de acordo com guias organizacionais de adaptação. Esses guias são instruções que possibilitam uma organização a adaptar a descrição de um processo padrão para atender a necessidades específicas [Iso/Iec, 2004].

Em organizações de alta maturidade é necessário que a definição de processos seja realizada através da escolha dos subprocessos que irão compor o processo [Chrissis et al., 2006; Softex, 2007]. Subprocessos, neste caso, são componentes definidos em um processo maior. Por exemplo, um processo de desenvolvimento típico de uma organização pode ser definido em termos de subprocessos, tais como desenvolvimento de requisitos, testes, revisões por pares, entre outros. Além de compor o processo com subprocessos menores, a escolha dos subprocessos deve ser feita com base em dados sobre a estabilidade e a capacidade desses subprocessos. Portanto, conhecer e controlar a estabilidade e capacidade dos (sub)processos é de fundamental importância para organizações de alta maturidade [Chrissis et al., 2006].

O processo estável pode ser definido como um processo previsível, cujo desempenho e variabilidade são conhecidos, permitindo elaborar estimativas que utilizam como base seu desempenho passado. Já o processo capaz é aquele cujo desempenho médio e variabilidade atendem ao especificado [Wheeler e Chambers, 1992].

Informações relacionadas à capacidade e estabilidade referentes aos subprocessos são muito importantes para a composição dos processos. Assim, um dos critérios a ser utilizado no momento de escolher os subprocessos pode ser a existência de dados sobre seu desempenho. Além disso, os objetivos de qualidade e desempenho do processo a ser definido também devem ser levados em consideração. Pode-se avaliar se os subprocessos apresentaram comportamento estável em utilizações anteriores, caso os contextos sejam comparáveis. É importante, também, considerar se os dados de desempenho dos subprocessos satisfazem aos objetivos de qualidade e desempenho do projeto que irá fazer uso do subprocesso [Chrissis et al., 2006].

Há diversos trabalhos na literatura que defendem que processos têm semelhanças com software, e que é possível aplicar métodos e técnicas de desenvolvimento de software na definição de processos [Osterweil, 1987]. Da mesma forma que há muitas analogias entre processos de software e produtos de software, podem ser feitas também analogias interessantes entre reutilização de processos de software e reutilização de produtos de software. KELLNER [1996] destaca que o conhecimento de técnicas de reutilização de produtos de software, tais como: arquiteturas povoadas com componentes reutilizáveis; gerenciamento de repositórios para armazenar, catalogar, procurar, acessar, etc. os ativos reutilizáveis; gerência de configuração de ativos reutilizáveis; entre outros; poderiam ser aplicados a processos de software.

Uma das formas reutilizar processos de software é através do uso de componentes de processo. Um componente de processo pode ser visto como um 
encapsulamento de informações e comportamentos de processo em um dado nível de granularidade [Gary e Lindquist, 1999]. Assim, um processo pode ser tratado como a integração de um conjunto de componentes de processo em diferentes níveis de granularidade [Fusaro et al., 1998]. Esses componentes podem fornecer apoio eficiente aos engenheiros de processo e equipes de projetos para a construção de modelos de processo e realização de ajustes dinâmicos no processo, o que contribui para a otimização e controle do processo [Ru-Zhi et al., 2005].

Processos de software completos podem ser decompostos em subprocessos. Uma vez que componentes de processo são decomposições de um processo inteiro, os subprocessos podem ser considerados componentes de processo que podem ser utilizados para compor um processo completo. Assim, componentes de processo podem ser utilizados para representar subprocessos conforme definido em modelos como o CMMI-DEV e o MPS.BR. Muitas vezes, também, utiliza-se o termo "elemento de processo" com um significado muito parecido ao de componente de processo. O CMMI-DEV, por exemplo, diz que um elemento de processo é a unidade fundamental (i.e., atômica) de definição de processos e descreve as atividades e tarefas necessárias para realizar o trabalho de forma consistente.

O conceito de arquitetura também é utilizado no contexto de processos de software. O CMMI-DEV define uma arquitetura de processos como a ordenação, interfaces, interdependências, e outros relacionamentos entre os elementos de processo em um processo padrão ou entre elementos de processos e processos externos. De forma simplificada, pode-se considerar que a arquitetura define o "esqueleto" que o processo deve possuir, determinando os principais elementos e como estes se relacionam, sem necessariamente definir como será seu detalhamento.

Linhas de produtos de software também podem ser adaptadas para o contexto de processos de software. Uma linha de produtos funciona como uma fábrica, que instancia produtos parecidos, cada um com um conjunto de características, por meio da composição de componentes existentes. Da mesma forma, é possível imaginar que se possam definir processos dessa maneira, ou seja, instanciar processos a partir de componentes de processos pré-existentes, sendo que cada instância teria um conjunto de características. Assim, linhas de processos são linhas de produtos cujos produtos são processos de software. A adoção de linhas de processos permite alavancar a reutilização de componentes de processo individuais para arquiteturas de processo completas, contendo diversos componentes de processo inter-relacionados. Esse conceito é recente na literatura e não há muitos trabalhos publicados sobre o assunto [Rombach, 2005; Washizaki, 2006; Barreto et al., 2008].

\section{Uma Abordagem para Definição de Processos Baseada em Reutilização}

Para contextualizar a etapa de componentização dos processos legados, descrevemos nesta seção uma visão geral da abordagem de definição de processos baseada em reutilização, em que a etapa se insere. Partes dessa abordagem já foram descritas em trabalhos anteriores [Barreto, 2007; Barreto et al., 2008] e apenas suas principais características e conceitos serão descritos aqui.

A abordagem para definição de processos baseada em reutilização busca facilitar a definição de processos através de sua composição a partir de componentes de 
processos reutilizáveis. Espera-se aumentar a produtividade da atividade (diminuição do esforço necessário para realizá-la); aumentar a qualidade e adequação dos processos gerados (reutilização do conhecimento de especialistas e de dados sobre utilização); facilitar a análise de estabilidade e desempenho dos subprocessos; representar variabilidades e semelhanças entre processos para potencializar a reutilização; entre outros. São utilizadas algumas técnicas comumente aplicadas na reutilização de produtos de software tradicional [Barreto et al., 2008].

Para permitir uma efetiva reutilização dos processos, é necessária a existência de uma ou mais bibliotecas de componentes reutilizáveis de processos. Essas bibliotecas armazenam componentes de processos, linhas de processos, conhecimento relacionado ao uso dos componentes, medições relacionadas ao uso dos componentes, entre outros. As bibliotecas podem ser utilizadas no momento da definição de processos para organizações ou projetos (definição com reutilização), onde componentes e demais itens reutilizáveis podem ser buscados e utilizados para compor processos. É necessário, também, um mecanismo de alimentação e evolução dessas bibliotecas de forma a oferecer aos projetos e organizações um conjunto útil e abrangente de itens reutilizáveis (definição para reutilização). A reutilização de processos pode ocorrer em diferentes cenários, tais como: (i) Instituições Implementadoras - podem necessitar definir processos, muitas vezes bastante semelhantes, para um conjunto de organizações diferentes. (ii) Organizações de Software - podem necessitar definir seus processos padrão, ou especializar esses processos para situações comuns na organização. Uma biblioteca de componentes reutilizáveis pode auxiliar nas definições tanto de processos padrão como dos processos definidos dos projetos. (iii) Projetos de Software - um projeto contribui para a biblioteca de componentes com informações de uso, medidas, solicitações de melhoria, entre outras informações relacionadas a real execução dos componentes de processo.

A Figura 1 ilustra o contexto da reutilização de processos em uma organização. A partir de requisitos gerais para processos de software, processos definidos anteriormente, processos disponibilizados pela Instituição Implementadora, modelos de maturidade, e outros requisitos de processos, a organização define e alimenta sua biblioteca com componentes de processo, linhas de processo, medidas e informações de uso desses itens, entre outros (definição para reutilização). Sempre que for necessário definir um processo para um projeto (definição com reutilização), os itens da biblioteca poderão ser (re)utilizados, com base nas necessidades específicas da situação, para compor o processo. Quando não existirem na biblioteca itens para atender a alguma necessidade específica, uma chamada ao processo de definição para reutilização pode ser feita. Assim, é possível enriquecer a biblioteca constantemente. A abordagem descrita neste artigo acontece na etapa de definição para reutilização, em situações onde os processos legados da organização são utilizados como insumo para essa atividade.

Para facilitar o entendimento deste artigo, é importante apresentar brevemente os principais conceitos que foram definidos em trabalhos anteriores [Barreto, 2007]:

- Elemento de Processo: Decomposição de processo em algum nível de granularidade. São definidos dois tipos: componentes de processo e atividades. Esses dois conceitos têm significados bastante similares, sendo que a principal diferença é que um componente é definido para reutilização. 
- Componente de Processo: Unidade básica de composição de processos. Definições de processo são sempre realizadas através da composição de diferentes componentes. Um componente é considerado algo relevante para: (i) ser reutilizado em outras definições de processo; (ii) ter sua estabilidade e desempenho analisado (iii) ser versionado; (iv) ter vários tipos de rastreabilidade estabelecidos; entre outros.

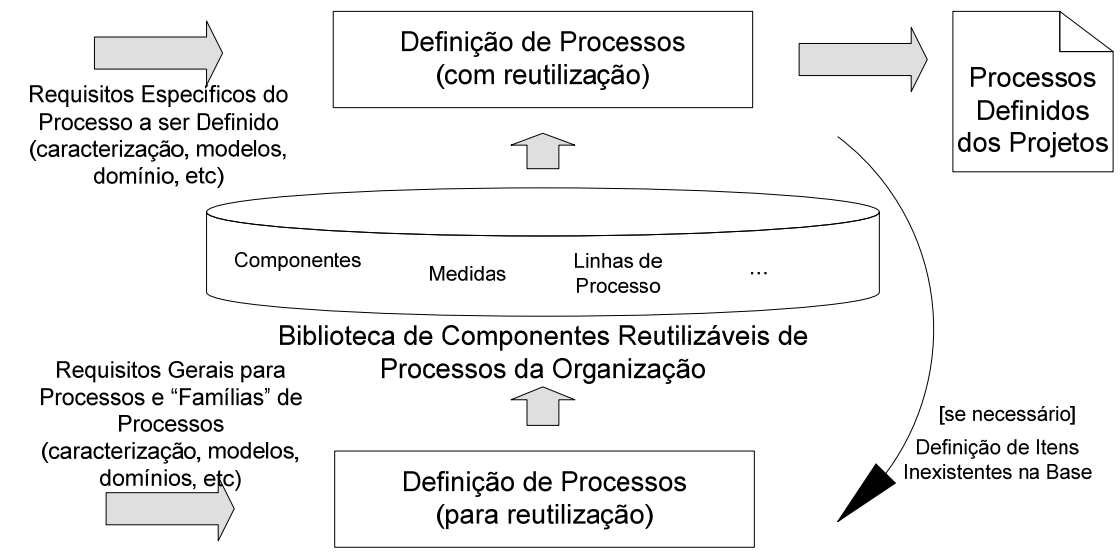

Figura 1 - Visão Geral da Abordagem de Reutilização na Organização

- Componente de Processo Concreto: Não admite qualquer variabilidade. Ou seja, precisa ser executado da forma que está descrito, sem qualquer variação. Em um componente concreto não há mais decisões a serem tomadas, tudo a ser definido no componente já o foi, e ele já pode ser utilizado diretamente em um projeto. Um componente concreto pode ser executado, medido, e controlado.

- Componente de Processo Abstrato: Componente que admite variabilidades, não estando vinculado a uma única forma de realização. Pode não possuir uma arquitetura interna, ou possuir uma que possua outros componentes abstratos. $\mathrm{O}$ termo "abstrato" foi escolhido pois esses componentes podem ser instanciados de diferentes maneiras, desde que atendam a um conjunto de restrições estabelecidas.

- Arquitetura de Processos: Representa uma espécie de fluxo de trabalho, podendo ser composta por componentes de processo, atividades ou qualquer combinação entre eles. A arquitetura de processos define um "esqueleto" que o processo deve possuir, determinando os principais elementos e como estes se relacionam, sem necessariamente definir como será o detalhamento desses elementos principais.

- Linha de Processos: Arquitetura de processos que possui variabilidades ou opcionalidades.

- Ponto de Variação: Elemento de uma arquitetura de processos que admite diversas estruturas internas, que podem ser realizadas por diversas variantes. $\mathrm{Ou}$ seja, um componente abstrato em uma arquitetura de processos é um ponto de variação.

- Variante: Uma das possíveis implementações de um ponto de variação. 
- Característica de Processo: Aplicação do conceito de característica (feature) de uma linha de produtos tradicional para o contexto de processos de software. Será vista como uma espécie de funcionalidade ou classificação que o processo deve possuir, por exemplo: Apoio ao nível G do MPS.BR, Paradigma Orientado a Objetos, entre outros. Restringe a utilização de componentes, definindo se um conjunto de componentes pode ou não ser utilizado. Podem ser utilizadas para representar diversos tipos de rastreabilidade.

A Figura 2 ilustra uma linha de processos exemplo. O primeiro componente da esquerda para a direita é o componente concreto "Planejar o Processo". Como esse componente é obrigatório (concreto), não admite variabilidades e precisará ser incluído no processo a ser definido através da linha de processos da forma como foi definido. $\mathrm{O}$ componente "Planejar o Projeto" teve sua arquitetura interna detalhada na figura. É possível observar que o componente "Definir Estimativas" é um componente abstrato, que desempenha o papel de ponto de variação. Assim, esse componente pode ser instanciado em um processo de três diferentes formas (suas variantes). Assim, no momento da definição do processo, o responsável pode escolher qual das variantes deve ser incluída no processo. Os componentes "Criar Planos" e "Obter Comprometimento" são também obrigatórios e devem ser incluídos nos processos que utilizarem a linha de processos da forma que estão, sem alterações. Por fim, o componente "Avaliação de Qualidade" representa um componente opcional que pode ou não ser incluído no processo que está sendo definido.

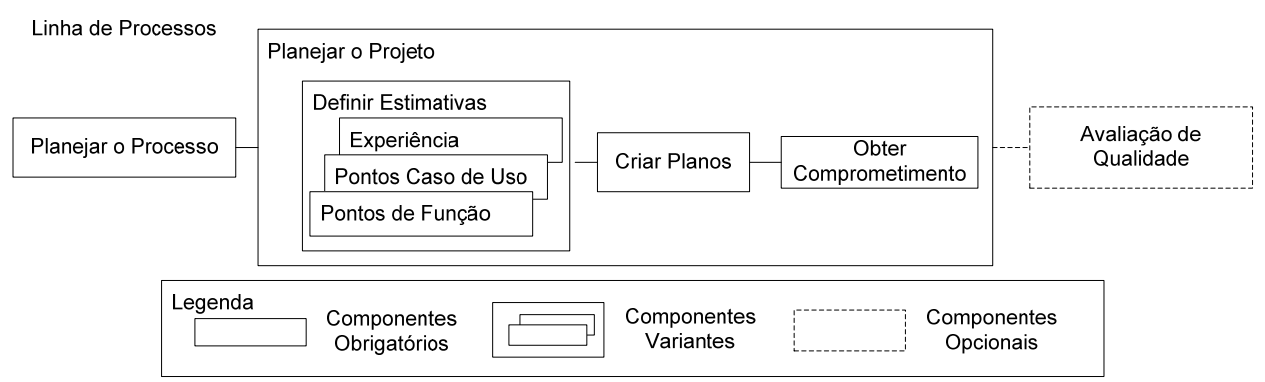

Figura 2 - Exemplo de Linha de Processos

\section{Componentizando Processos Legados}

Como dito na seção 2, em organizações de mais alta maturidade é necessário que a definição de processos seja realizada através da escolha dos subprocessos que irão compor o processo em si [Chrissis et al., 2006]. Além de compor o processo com base em subprocessos, a escolha dos subprocessos deve ser feita com base em seus dados de estabilidade e capacidade. Para atingir esses objetivos, organizações podem partir de uma situação em que já existam processos e ativos de processo definidos e em uso. Portanto, na preparação para a adoção das práticas de alta maturidade, uma fase inicial de componentização dos processos "legados" é importante para que os processos assumam uma estrutura que facilite sua reutilização, análise de estabilidade e capacidade, simulação, dentre outras práticas requeridas pelos níveis mais altos de maturidade.

Para ilustrar como a componentização de processos legados pode ser realizada em uma situação real e facilitar seu entendimento, descreveremos ao longo do texto um exemplo de utilização da proposta no contexto dos processos da Área de Qualidade do LENS (Laboratório de Engenharia de Software) da COPPE/UFRJ. A Área de Qualidade 
do LENS realiza, dentre outras atividades, projetos de desenvolvimento de software, tanto no contexto das teses e dissertações quanto em parceria com a indústria. É utilizado um processo padrão de desenvolvimento de software, que é adaptado para cada projeto específico. Em 2008, foi avaliada com sucesso no nível E de maturidade do MR-MPS e pretende, no futuro, ser avaliada no nível A do mesmo modelo. Assim, os processos existentes atualmente precisam ser ajustados e novos processos precisam ser definidos para se atingir a alta maturidade.

Para componentizar processos, quatro etapas principais são sugeridas, como esquematizado na Figura 3 e detalhadas nas seções a seguir:

1.Definir componentes de processos;

2.Definir características de processos;

3.Definir uma linha de processos;

4.Aprovar inclusão de elementos reutilizáveis na biblioteca de componentes.

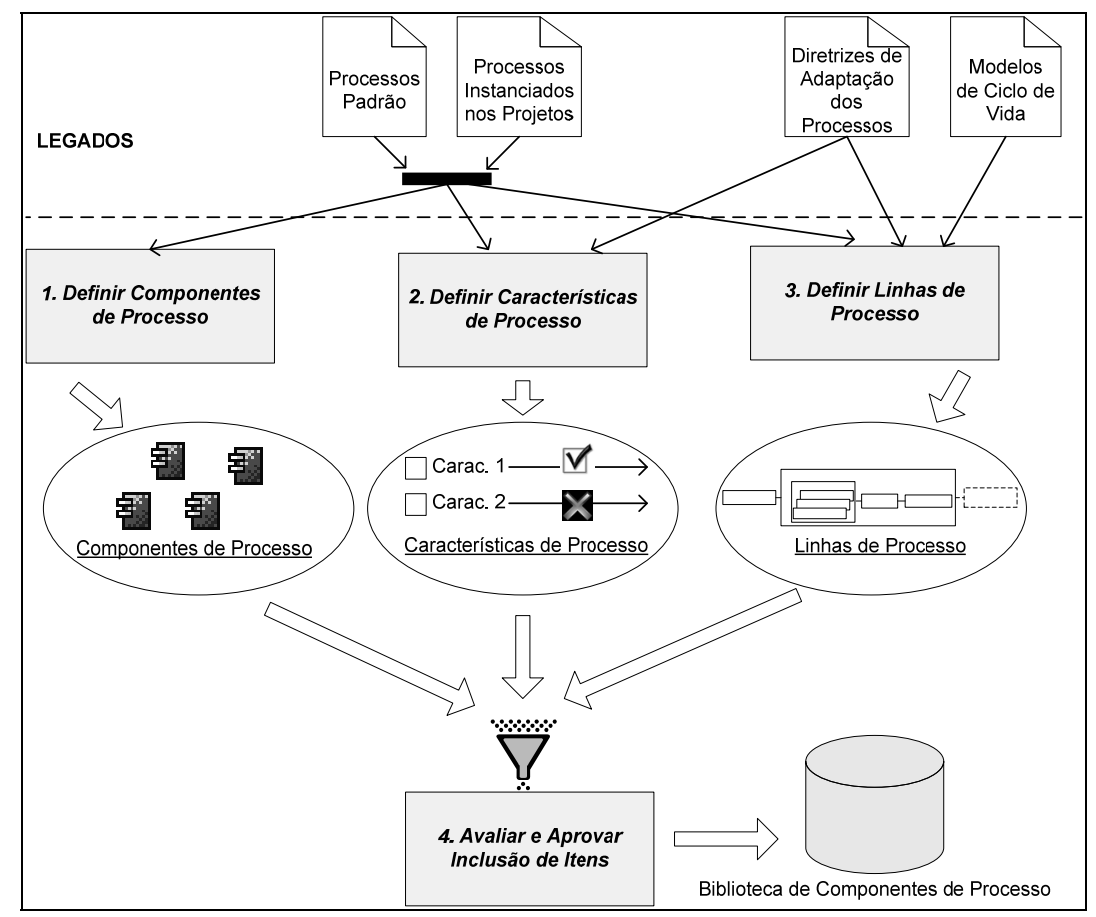

Figura 3 - Componentizando Processos Legados

\section{1 - Definição de Componentes de Processo}

O objetivo desta etapa é definir componentes de processo que representem subprocessos potencialmente relevantes para o contexto da reutilização de processos ou da alta maturidade. Nesta etapa devem ser considerados o processo padrão e todos os processos já definidos para projetos da organização.

Deve ser feita análise de todos os processos definidos de projetos da organização. Estes processos podem conter alterações em relação ao processo padrão, que são boas fontes de informação para a componetização. Devem ser detectadas as "partes" do processo padrão que sempre estiveram presentes nos projetos, e quais sofreram variação. As "partes" que sofreram variação indicam que esses são pontos de 
variação do processo, e que, portanto, podem ser realizadas de mais de uma maneira. Todas essas partes variáveis devem ser consideradas componentes de processo abstratos, que indicam a possibilidade de diversas realizações de um subprocesso. Devem também ser criados componentes concretos para cada possível maneira de realização (variantes).

Considerando os processos da Área de Qualidade do LENS, foram analisados os documentos de justificativas de alteração no processo padrão (artefato gerado durante o planejamento dos processos dos projetos) de 12 projetos. Foram detectadas algumas alterações comuns nos processos que se transformaram em componentes, por exemplo: (i) Exclusão de atividades de modelagem, tais como: elaboração de diagramas de seqüência, de diagramas complementares ou de projeto de banco de dados; (ii) Inclusão de atividades de monitoração do projeto e de gerência de planos de ação; (iii) Inclusão de atividades de prototipação; (iv) Inclusão de atividades de construção de classes em $\mathrm{C}++$ e conversão para Java. $\mathrm{O}$ fato de alguns grupos de atividades terem sido excluídos ou incluídos no processo padrão indica que esses grupos representam variações no processo que podem ser representadas como componentes para facilitar a reutilização. Assim, foram criados componentes abstratos para indicar as possibilidades de variação e componentes concretos para representar cada uma das instanciações possíveis.

A Figura 4 ilustra uma dessas situações. Nesta figura, utilizamos uma notação adaptada do diagrama de componentes de processo sugerido pelo SPEM [Omg, 2008], indicando os componentes de processo, seus tipos (através dos estereótipos: "concreto" ou "abstrato"), seus parâmetros de entrada e saída (artefatos consumidos e produzidos os parâmetros das variantes são os mesmos do componente abstrato e por isso foram omitidos), além das relações indicando que um componente é uma variante de outro. No exemplo da Figura 4, temos o componente abstrato "Elaborar Modelo de Análise e Projeto" e a ele associadas três variantes, definidas a partir da análise das adaptações realizadas até o momento no processo padrão. Portanto, será possível realizar a elaboração do modelo de análise e projeto de três formas distintas nos projetos, dependendo do componente escolhido na definição de seu processo.

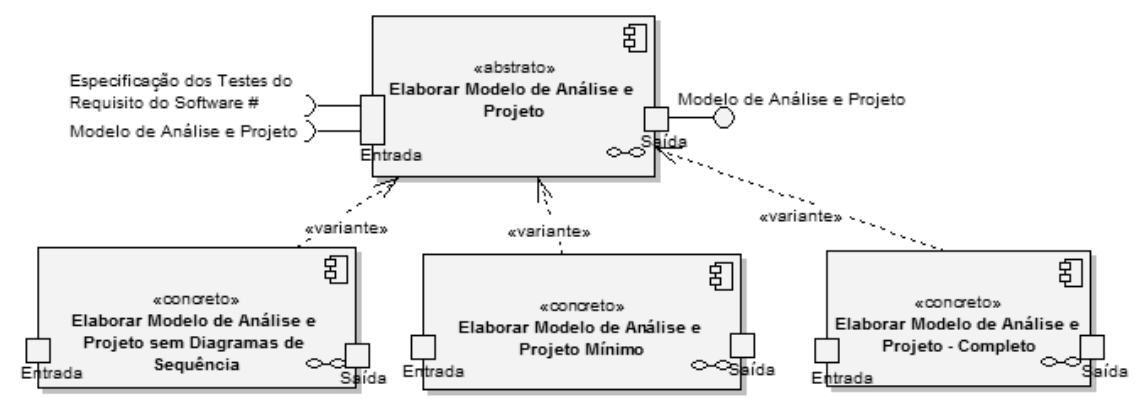

Figura 4 - Exemplo de Componente Abstrato e suas Variantes

Depois disso, deve-se buscar identificar agrupamentos de atividades que: (i) estejam em um nível de detalhamento tal que possam ser facilmente reutilizadas em outras definições; (ii) sejam potencialmente relevantes para análises de capacidade e estabilidade; ou (iii) já possuam dados de utilização, de forma a tornar possível a utilização de dados passados nas análises de estabilidade e capacidade futuras. Por exemplo, em processos que são estruturados em macro-atividades, essas macroatividades são boas candidatas a se tornarem componentes de processo que atendem aos 
critérios citados neste passo. Aqueles componentes que representem agrupamentos de elementos de processo que nunca tenham sofrido alteração e que aparentemente não tenham esse potencial devem ser considerados componentes concretos. Nos demais casos, os componentes devem ser abstratos. Esse passo pode ser aplicado recursivamente para cada componente definido, uma vez que pode haver ainda a necessidade de componentes em uma granularidade mais fina. Por exemplo, no caso de ser definido um componente "Planejar o Projeto", mesmo que esse componente seja importante para a reutilização e para análises do processo, ainda há a possibilidade de detalhar sua estrutura interna em outros componentes relevantes, como "Estabelecer Estimativas" ou "Planejar os Recursos do Projeto", que podem ser importantes pontos de variação no processo. Vale salientar que esse nível de detalhamento será muito dependente da estrutura do processo legado e dos objetivos que se quer alcançar com a componentização.

Considerando novamente os processos da Área de Qualidade do LENS, todas as macro-atividades originaram componentes de processo. Essa escolha foi feita devido ao bom nível de granularidade e devido à existência de medidas relacionadas às macroatividades. Dentro das macro-atividades, outros componentes foram também definidos. Por exemplo, a macro-atividade "Especificação de Requisitos" envolvia atividades de especificação dos requisitos de software, especificação de casos de testes para os requisitos de software e até cálculo do tamanho de software após definição dos requisitos. Neste caso percebeu-se claramente que havia necessidade de quebrar o componente "Especificação de Requisitos" em outros componentes de granularidade mais fina. Neste detalhamento vale salientar que nem todas as atividades precisam ser consideradas componentes de processo. O mesmo componente "Especificação de Requisitos" foi especificado com algumas atividades em seu interior, como "Criar Matriz de Rastreabilidade", que foi considerada muito simples e estática para justificar um componente de processo.

Além da abordagem anterior, que pode ser considerada reativa, sendo executada função de eventos que já aconteceram, uma abordagem proativa também pode ser adotada, visando antecipar necessidades futuras. Nessa abordagem se deve buscar identificar elementos de processo no processo legado que, mesmo que não tenham ainda sofrido alguma variação nas instanciações de processo, possuam grande potencial de variação. Ou seja, claramente possam ser realizados de diversas formas no contexto dos projetos da organização. Talvez a variação não tenha ainda ocorrido exatamente pelo fato de as definições e orientações sobre como realizá-las não estarem presentes. Exemplos são atividades que podem ser realizadas de acordo com uma ou outra técnica específica, como atividades de estimativas ou de avaliação de qualidade. No caso dos processos da Área de Qualidade do LENS, foram identificados alguns desses casos. Por exemplo, as atividades de cálculo do tamanho do software originaram componentes abstratos, com pelo menos dois componentes concretos associados, um para o cálculo com Pontos de Função e outro com Pontos de Caso de Uso.

Vale ressaltar que as informações originadas das atividades ou outros elementos de processos que originem os componentes devem ser refletidas neste. Ou seja, medidas, critérios de entrada e saída, artefatos consumidos e produzidos e outras informações associadas devem ser adaptadas para serem aplicáveis não a uma única atividade, mas ao componente como um todo. Por exemplo, se uma macro-atividade é 
considerada um componente e existiam métricas associadas a ela, as mesmas métricas podem passar a ser associadas ao componente, mas em outros casos pode ser necessária alguma adaptação.

É de se esperar que com o tempo, ações que eram realizadas apenas de uma maneira (e provavelmente tenham originado um componente concreto) venham a sofrer alterações. Com isso, um componente abstrato mais geral pode ser criado e o componente concreto anterior pode se tornar apenas uma das variantes do novo componente abstrato. Assim, a componentização inicial pode ser bastante evoluída a partir da constante melhoria dos processos.

Para finalizar, descrevemos um breve resumo das questões que, quando respondidas positivamente, podem levar um elemento de processo (e.g., atividade, macro-atividade, subprocesso) legado a se tornar um componente de processo: (i) $\mathrm{O}$ elemento está em um nível de detalhamento tal que possa ser facilmente reutilizado? (ii) O elemento é potencialmente relevante para análises de capacidade e estabilidade? (iii) O elemento já possui dados de utilização, de forma possibilitar a utilização de dados passados nas análises de estabilidade e capacidade futuras? (iv) O elemento já sofreu variação em algum dos processos definidos de projetos (i.e., foi excluído, incluído ou modificado)? (v) O elemento, mesmo não tendo sofrido variação nas instanciações de processo, possui grande potencial de variação, ou seja, claramente pode ser realizado de diversas formas no contexto dos projetos da organização?

\section{2 - Definição de Características de Processo}

Esta etapa da componentização de processos envolve a definição de características de processo. Através dessas características é possível registrar diferentes tipos de rastreabilidade para componentes de processo. As características de processo restringem quais componentes podem ser escolhidos durante a definição de processos. Assim, o objetivo desta etapa é determinar variados fatores que podem influenciar na escolha de um ou outro componente de processo no momento de uma definição de processos e registrar esse conhecimento através das características de processos.

Uma das principais fontes de informação para a definição de características de processo são as diretrizes de adaptação dos processos padrão da organização. Nessas diretrizes estão estabelecidas as situações em que uma ou outra adaptação no processo pode ocorrer. Assim, é possível definir características que representem as diferentes possibilidades de adaptação do processo e associar a elas os componentes de processo que devem ou não ser escolhidos, uma vez que a característica seja selecionada, como exemplificado na Figura 5. Nesta figura, é possível ver algumas diretrizes de adaptação de processos, as características que poderiam ser criadas a partir delas e as relações entres as características e os componentes de processo (selecionando uma ou outra característica um componente pode ou não ser escolhido). De certa forma, essa estratégia consiste em promover um conhecimento explicito, porém indireto, para uma entidade de primeira classe: uma característica. Sendo assim, a característica pode ser vista como um encapsulamento de todo um conjunto de possibilidades de adaptação de um processo, viabilizando a sua automação.

Além disso, é importante considerar as peculiaridades do processo legado para se registrar esse conhecimento como características de processo. Um exemplo nos processos da Área de Qualidade do LENS é a aderência ao nível E do MR-MPS, que já 
existe nos processos legados. Assim, podemos associar os componentes a diversas características relacionadas a modelos de maturidade, tais como: Aderência ao Nível G do MR-MPS, Aderência ao Nível E do MR-MPS, Aderência ao Nível 2 do CMMI. Dependendo da modelagem de características escolhida, é possível associar os componentes a características de vários outros tipos, como: disciplinas (planejamento de projetos, requisitos, testes), técnicas e métodos utilizados (análise de pontos de função, inspeções), paradigmas de desenvolvimento (estruturado, orientado a objetos). Assim, é importante registrar as características já atendidas pelos processos legados.

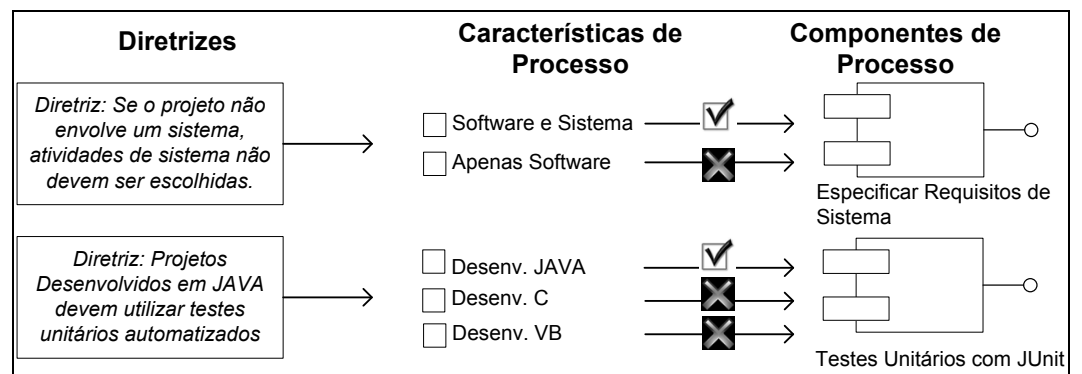

Figura 5 - Exemplo de Utilização das Diretrizes de Adaptação do Processo Padrão para Definir Características de Processo

Um checklist que contenha uma série de características comuns e seus respectivos tipos pode ser usado neste momento para que nenhuma classificação do processo legado seja esquecida, como exemplifica a Figura 6.

\begin{tabular}{|c|c|c|c|c|c|c|c|}
\hline \multicolumn{8}{|c|}{ Caracterísicas de Processo } \\
\hline \multicolumn{8}{|c|}{ Modelos de Maturidade } \\
\hline MPS-BR-G & MPS-BR-F & MPS-BR-E & MPS-BR-D & MPS-BR-C & MPS-BR-B & CMMI-2 & ... \\
\hline \multicolumn{8}{|c|}{ Tipos de Software } \\
\hline Soft. WEB & Soft. Plataf & ma Alta & DW & $\ldots$ & & & \\
\hline \multicolumn{8}{|c|}{ Paradigmas de Desenvolvimento } \\
\hline Orientação & Objetos & Orientaçầ & Aspectos & Estruturado & & $\ldots$ & \\
\hline \multicolumn{8}{|c|}{ Domínios de Aplicação } \\
\hline Financeiro & Bancário & Portuário & Militar & Siderúrgia & Automotivo & &.. \\
\hline
\end{tabular}

Figura 6 - Exemplo de Checklist de Características de Processo

Algumas organizações, como é o caso da Área de Qualidade do LENS, já possuem um conjunto de fatores que devem ser considerados para caracterizar um projeto e essa informação é usada nas definições de processo. Exemplos desses fatores são: o tipo de software a ser desenvolvido, o paradigma de desenvolvimento a ser utilizado, o domínio de aplicação, além de outros fatores ligados ao problema, ao produto e ao projeto. Esse conjunto de fatores, quando existente, pode ser uma ótima fonte de informação para a definição de características de processo.

\section{3 - Definição de Linhas de Processo}

Uma vez que componentes de processo estejam definidos e classificados para a organização, é importante considerar alternativas de estruturas para relacionar e ordenar esses componentes de forma a poderem ser usados em um processo. Para isso, podem ser usadas arquiteturas ou linhas de processo, que são definidas nesta etapa.

Linhas de processo também podem estar associadas a características de processo, de forma que durante uma definição de processos, a escolha da linha de processos também possa ser feita através da seleção de características. Assim, o 
primeiro passo na definição das linhas de processo pode ser a escolha de quais características a linha de processos deve atender. Fazendo desta forma, as características escolhidas servirão como requisitos ou diretrizes que devem ser observados ao longo da definição da linha, além de uma forma de registro de rastreabilidade. No entanto, podese optar também por associar as características ao final, depois que a linha estiver definida, utilizando-as apenas para registrar a aplicabilidade da linha de processos.

Para seguir na definição das linhas, uma abordagem top-down é sugerida, partindo da determinação do "esqueleto" que os processos gerados a partir dela terão. Um bom ponto de partida são os modelos de ciclo de vida utilizados pela organização. Cada um dos modelos utilizados pode ser considerado uma linha de processos diferente (ou pelo menos um primeiro passo na definição de uma linha de processo mais complexa), em que o relacionamento entre os componentes e a ordem de execução estarão definidos. Também é possível determinar quais componentes serão repetíveis, considerando as partes iterativas do modelo de ciclo de vida. A Figura 7 exemplifica parte de uma linha de processos, definida a partir dos processos legados da Área de Qualidade do LENS, considerando o modelo cascata. No exemplo é exibida apenas a parte referente à primeira fase do processo legado, pois é bem simples e através dela é possível ilustrar os principais conceitos. Nesta primeira fase do processo, houve pouca variação nas instanciações, o que fez com que quase todos os componentes definidos fossem concretos. As atividades relacionadas à estimativa e ao cálculo do tamanho do software apresentavam bom potencial de variação, por isso foram definidas como componentes abstratos, com variantes associadas. Não havia elementos opcionais nem repetíveis considerando os processos legados e isso foi refletido na linha de processos.

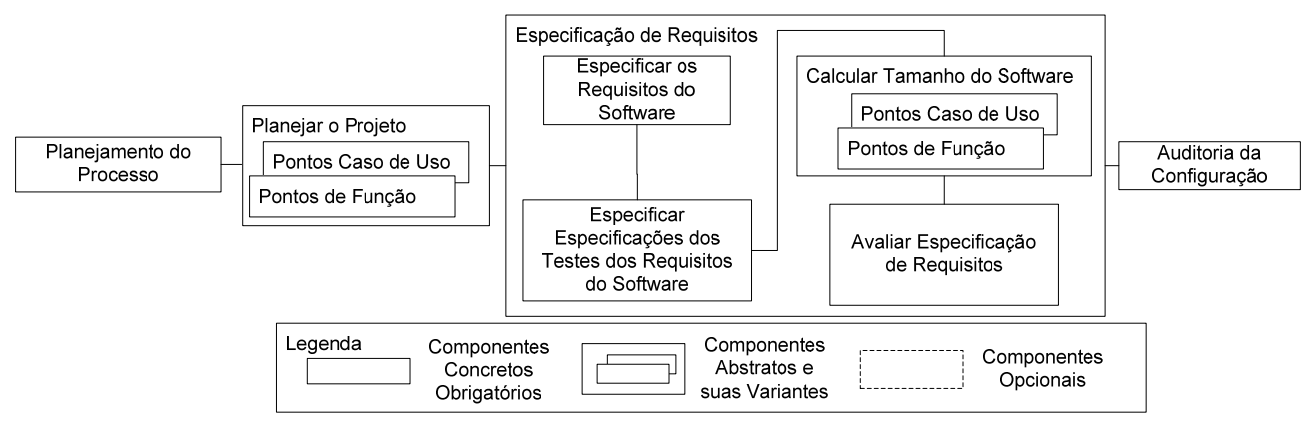

Figura 7 - Exemplo de Linha de Processos

Para detalhar as linhas de processo definidas, ou até definir outras, devem ser consideradas, também, as diretrizes de adaptação do processo padrão. Essas diretrizes podem auxiliar principalmente na definição dos elementos opcionais e pontos de variação da linha de processo. As adaptações realizadas no processo até o momento, analisadas na primeira etapa descrita (seção 4.1), também podem ser utilizadas para esse fim. Todas essas informações, juntamente com as características de processo, podem ser importantes na determinação de quais variantes poderão ser escolhidas em cada ponto de variação da linha. Essa escolha é bastante semelhante à ilustrada na Figura 5.

\section{4 - Avaliação e Aprovação dos Elementos Reutilizáveis}

O último passo envolve a avaliação, pelos stakeholders relevantes, dos componentes, características e linhas de processo candidatos, antes de serem incorporados à base de componentes da organização. 
Neste passo podem ser definidas ou utilizadas diretrizes organizacionais para aceitação de elementos reutilizáveis de processo (o processo Gerência de Reutilização do MR-MPS pode ser considerado). Além disso, é importante que os envolvidos concordem que os itens são relevantes e vão permitir a adequada reutilização de processos na organização. Também devem ser descartadas as possíveis variantes que violem as diretrizes de adaptação do processo padrão existentes na organização. Os itens aprovados podem, então, ser armazenados na biblioteca de componentes. Com isso, é de se esperar que já seja possível realizar a primeira definição de processos com base na biblioteca de componentes da organização.

\section{Conclusão}

Neste trabalho, foi apresentada uma abordagem para componentização de processos legados de software. Esta proposta faz parte de uma abordagem mais ampla de definição de processos baseada em reutilização, que por sua vez faz parte de um conjunto de pesquisas relacionadas a práticas de alta maturidade que vêm sendo feitas na COPPE/UFRJ. Acredita-se que a utilização de componentes, características e linhas de processo na definição de processos de software facilita e viabiliza a reutilização de processos, além de facilitar o alcance de vários resultados esperados pelos modelos de maturidade relacionados a níveis mais altos. Acredita-se, ainda, que a definição dos passos a serem seguidos e de diretrizes para guiar a criação de elementos reutilizáveis de processos a partir de processos e ativos de processos legados seja útil para organizações desejando tornar seus processos mais reutilizáveis.

Após o exercício de componentização realizado, foi possível perceber que tornar os processos reutilizáveis é uma tarefa custosa, pois muitas situações diferentes precisam ser previstas e explicitadas através de componentes, linhas e características. Acreditamos que o apoio ferramental amenize a situação, mas que o custo inicial para formalizar o conhecimento sobre os processos seja compensado pela diminuição do esforço necessário para definir os processos no futuro. Nos casos em que a Instituição Implementadora de Processos fornece os elementos reutilizáveis para as organizações, os ganhos tendem a ser ainda maiores. Percebemos também que as etapas de componentização não precisam ocorrer isoladamente, pois ao longo da execução de uma das etapas (ex.: definição de linhas de processo), pode-se perceber a necessidade de se criar ou melhorar algum item criado em outra etapa (ex.: definição de componentes). Também se constatou a necessidade de criação de um checklist para melhor avaliar a componentização realizada, o que já começou a ser feito.

Como próximos passos é possível citar a componentização de mais processos legados existentes, considerando também o contexto em que a COPPE/UFRJ atua como Instituição Implementadora de Processos, o que já foi iniciado. Além disso, protótipos das ferramentas de apoio estão em desenvolvimento. Esses protótipos facilitarão a aplicação da abordagem proposta e da reutilização de processos como um todo.

\section{Referências}

Barreto, A., 2007, Uma Abordagem para Definição de Processos de Software Baseada em Reutilização, Exame de Qualificação para o Doutorado, Programa de Engenharia de Sistemas e Computação, COPPE/UFRJ, Rio de Janeiro, Brasil. 
Barreto, A., Murta, L., Rocha, A.R., 2008, "Software Process Definition: a Reuse-based Approach". In: XXXIV Conferencia Latinoamericana de Informática (CLEI'08), Santa Fe, Argentina, Setembro de 2008.

Chrissis, M.B., Konrad, M., Shrum, S., 2006, CMMI: Guidelines for Process Integration and Product Improvement, 2nd ed. Nova York, Estados Unidos, Addison-Wesley.

Fusaro, P., Visaggio, G., Tortorella, M., 1998, "REP - ChaRacterizing and Exploiting Process Components: Results of Experimentation". In: Working Conference on Reverse Engineering, pp. 20-29, Honolulu, United States, October.

Gary, K.A., Lindquist, T.E., 1999, "Cooperating Process Components". In: International Computer Software and Applications Conference (COMPSAC), pp. 218-223, Phoenix, United States, October.

Humphrey, W.S., 1989, Managing the Software Process, 1st ed. Boston, Estados Unidos, Addison-Wesley.

Iso/Iec, 2004, "Information Technology - Software Process Assessment", v. ISO/IEC 15504.

Kellner, M.I., 1996, "Connecting Reusable Software Process Elements and Components". In: 10th International Software Process Workshop, pp. 8-11, Dijon, France, June.

Omg, 2008, "SPEM - Software Process Engineering Metamodel", Object Management Group, v. SPEM.

Osterweil, L., 1987, "Software Processes Are Software Too". In: International Conference on Software Engineering, pp. 2-13, Monterey, Estados Unidos, April.

Reis, R.Q., 2002, APSEE-Reuse: Um Meta-Modelo para Apoiar a Reutilização de Processos de Software, Tese de D.Sc., PPGC, UFRGS, Porto Alegre, Brasil.

Rombach, H.D., 2005, "Integrated Software Process and Product Lines". In: International Software Process Workshop, pp. 83-90, Beijing, China, May.

Ru-Zhi, X., Tao, H., Dong-Sheng, C., et al., 2005, "Reuse-Oriented Process Component Representation and Retrieval". In: International Conference on Computer and Information Technology, pp. 911-315, Shangai, China, September.

Softex, 2007, "MPS.BR - Melhoria de Processo do Software Brasileiro, Guia Geral (v1.2)", SOFTEX - Associação para Promoção da Excelência do Software Brasileiro, v. SOFTEX.

Washizaki, H., 2006, "Building Software Process Line Architectures from Bottom Up". In: Product-Focused Software Process Improvement, pp. 415-421, Amsterdam, Netherlands, June.

Wheeler, D.J., Chambers, D.S., 1992, Understanding Statistical Process Control Knoxville, Estados Unidos, SPC Press. 